\title{
The Impact of the Preoperative Status on the Short-term Outcomes After Exenteration and Pelvic Reconstruction
}

\author{
NICOLAE BACALBASA ${ }^{1,2,3}$, IRINA BALESCU ${ }^{4}$, MIHAELA VILCU $^{1,2}$, SIMONA DIMA $^{3}$ and IULIAN BREZEAN ${ }^{1,2}$ \\ 1 "Carol Davila" University of Medicine and Pharmacy, Bucharest, Romania; \\ 2 "I. Cantacuzino" Clinical Hospital, Bucharest, Romania; \\ 3 “Fundeni” Clinical Institute - Center of Excellence in Translational Medicine, Bucharest, Romania; \\ 4 “Ponderas" Academic Hospital, Bucharest, Romania
}

\begin{abstract}
Background/Aim: The aim of this study was to analyse the influence of the preoperative status on the perioperative outcomes of patients submitted to pelvic reconstructions after exenteration. Materials and Methods: Between January 2017 and December 2018, pelvic exenteration was performed in 86 cases; patients were classified according to their age, nutritional status and association of reconstructive surgery. Results: The median age was 56 years, while the median level of serum albumin was $3.6 \mathrm{~g} / \mathrm{dl}$. Reconstructive surgery was more frequently performed in younger patients, while the rate of postoperative complications was similar between the two groups, while the rate of postoperative complications was significantly higher among cases with lower serum albumin levels. Conclusion: Reconstructive surgery should be performed in selected patients. Elderly cases as well as those presenting a poorer nutritional status are at higher risk of developing postoperative complications.
\end{abstract}

Although initially patients diagnosed with advanced stage pelvic malignancies were considered as incurable and were addressed to palliative medical treatment, since Brunschwig reported the first series of cases submitted to pelvic exenteration, attention has been focused on improving the outcomes of these patients (1). Therefore, reconstructive procedures have been implemented for both urinary and digestive tract alone or in association with vaginal or perineal reconstructions. However, performing these techniques usually associates with a higher number of

This article is freely accessible online.

Correspondence to: Irina Balescu, "Ponderas" Academic Hospital, Bucharest, Romania. Tel: +40 724077709, e-mail: irina.balescu@ ponderas-ah.ro

Key Words: Pelvic reconstruction, clinical status, biological status, perioperative outcomes. anastomoses and, consequently, a higher risk of postoperative complications. The aim of this study was to analyse the clinical and biological factors which might influence the risk of postoperative complications in patients submitted to pelvic reconstructions after exenteration.

\section{Materials and Methods}

Between January 2017 and December 2018, pelvic exenteration with curative intent for locally advanced gynecological malignancies was performed in 86 cases. These patients were classified according to their age (younger than 60 years and elderly than 60 years), nutritional status (good nutritional status - albumin level of at least $3.5 \mathrm{~g} / \mathrm{dl}$, and poor nutritional status - albumin level lower than $3.5 \mathrm{~g} / \mathrm{dl}$ ), and the association with a reconstructive procedure. Postoperative complications were classified according to Clavien-Dindo scale (2), while statistically significant results were considered if the $p$-value was lower than 0.05 .

\section{Results}

The median age of patients at the time this study was conducted, was 56 years (range $=36-76$ years). Among these cases, 56 were younger than 60 years while the remaining 30 cases were elderly. In 59 cases, the origin of the tumor was at the level of the uterine cervix, in 18 cases the origin was vulvar, in six cases the primary tumor was located at the level of the uterine body, while in the remaining three cases the primary tumor was ovarian cancer. In 63 cases surgery was performed for primary lesions, while in the remaining 23 cases pelvic exenteration was needed for recurrent disease. In all cases, surgery with radical intent was attempted. Regarding preoperative albumin level, in 63 cases it was higher than $3.5 \mathrm{~g} / \mathrm{dl}$, while in the remaining 23 cases this level was lower, the median level being $3.6 \mathrm{~g} / \mathrm{dl}$ (range=2.9-3.8 g/dl).

Details regarding the preoperative and intraoperative characteristics classified according to patients' age and nutritional status are shown in Tables I and II. 
As it can be observed from these data, elderly patients were more likely to develop vulvar cancer, while younger than 60-year-old patients were more likely to be submitted to pelvic exenteration for cervical cancer. Elderly patients were more likely to present associated comorbidities; however, the only statistically significant association was found between older age and arterial hypertension $(p=0.032)$. As for the type of disease, primary versus recurrent, although younger patients were more frequently diagnosed with primary tumors, this fact did not reach statistical significance. When it comes to the impact of the nutritional status defined by the serum level of albumin, it was significantly associated with the body mass index (BMI) value, while no other studied parameter was statistically significant.

Data regarding the intraoperative and postoperative outcomes of the patients stratified according to their age are presented in Table III. Reconstructive surgery was more frequently associated with younger patients and consisted of continent urinary tract conduits, colorectal anastomoses and vaginal/ perineal reconstructions. However, the rates of postoperative complications requiring reintervention (grade 4-5 according to Clavien-Dindo scale) were similar between the two groups. Three patients from the first age group and two cases from the second group died to septic shock within the first postoperative month.

In regards to the influence of the nutritional status on the intraoperative and postoperative outcomes, patients presenting a lower preoperative albumin level were more often submitted to incontinent urinary and digestive reconstructions and benefitted less from vaginal/perineal reconstructions. Even so, major complications (ClavienDindo grade 3-5) were significantly more common among patients with lower albumin levels (Table IV).

As for the impact of the preoperative BMI on choosing the type of reconstructive surgery, obese patients (defined by a BMI value $>30 \mathrm{~kg} / \mathrm{m}^{2}$ ) were less frequently submitted to reconstructive surgery; however, they developed more frequently complications. Among the 26 cases of the obese patients, urinary tract reconstructions were performed in three cases, digestive tract reconstructions were performed in six cases and vaginal/perineal reconstructions were performed in two cases. Postoperative complications were encountered in 13 cases ( $50 \%$ of cases) and consisted of Clavien-Dindo grade 2 complications in seven cases (pneumonia in six cases and wound infection in four cases), grade 3 complications in two cases (pelvic abscesses which were managed in a percutaneous manner in both cases) and grade 4 complications in four cases. In these four cases, reoperation was needed for hemoperitoneum in three cases and respectively for urinary leak in one case. However, none of these cases died within the first operative month.

\section{Discussion}

Performing extended pelvic resections in order to achieve an R0 resection for locally advanced gynecological malignancies remains a demanding procedure which requires various surgical gestures including digestive, urinary and even perineal resections followed in certain cases by reconstructive procedures. Prior to Brunschwig's era, patients will locally-advanced pelvic malignancies were considered as terminal and, in consequence, were solely addressed to the oncologists for palliative care, while their survival was minimal. Once Brunschwig reported the first successful series of patients submitted to pelvic exenteration and demonstrated that this procedure could improve the quality of life even if it is performed with palliative intent, it has been widely implemented and submitted to permanent changes (1). Therefore, pelvic exenteration was transformed in time into a curative, radical procedure, with improved rates of 5-year survival $(3,4)$.

Association of new concepts such as total mesorectal excision and total mesometrial excision as well as of the adjuvant radiation therapy leaded to increased rates of survival, with lower rates of pelvic recurrences (3-5). In this context, attention was focused on improving the quality of life and creating various techniques of reconstructions. Therefore, nowadays a patient submitted to pelvic exenteration is no longer the equivalent of a permanent carrier of digestive and urinary external derivations or ostomies $(3,6,7)$. Moreover, introduction of different techniques of pelvic reconstruction is associated with the interposition at the level of the pelvic, denuded, hypoxic floor of healthy, well vascularized tissues, impeding in this way the development of postoperative complications such as pelvic abscesses, severe small bowel complications or empty pelvis syndrome. Moreover, in cases in which the anatomical condition does not allow these types of reconstructions, artificial materials might be used with satisfactory results (8-10).

However, it should not be omitted that pelvic and perineal reconstructions using synthetic materials might induce in certain cases the development of further complications such as extrusion, infection or foreign body reactions (11). Therefore, certain authors proposed the use of bioprosthetic mesh materials such as human acellular dermal matrix, with interesting results (12).

Association of pelvic reconstructions after exenteration should be performed especially in cases with a good clinical and biological status in order to provide an adequate healing of the wounds without increasing the risk of perioperative morbidity and mortality, while providing proper function and anatomy. However, the decision of performing a reconstructive procedure should be taken only after discussing with both the patient and the family about the supplementary perioperative risks which are associated with 
Table I. Patients' characteristics classified according to patients' age.

\begin{tabular}{|c|c|c|c|}
\hline Characteristics & Age $<60$ years ( 56 cases) & Age $>60$ years ( 30 cases) & $p$-Value \\
\hline \multicolumn{4}{|l|}{ BMI } \\
\hline$<18 \mathrm{~kg} / \mathrm{m}^{2}$ & $12(21.4 \%)$ & $2(6.7 \%)$ & 0.056 \\
\hline $18-30 \mathrm{~kg} / \mathrm{m}^{2}$ & $34(60.7 \%)$ & $12(40.0 \%)$ & 0.753 \\
\hline$>30 \mathrm{~kg} / \mathrm{m}^{2}$ & $10(17.9 \%)$ & $16(53.3 \%)$ & 0.022 \\
\hline \multicolumn{4}{|l|}{ Comorbidities } \\
\hline Hypertension & $4(7.1 \%)$ & $16(53.3 \%)$ & 0.032 \\
\hline Diabetes mellitus & $8(14.3 \%)$ & $6(20.0 \%)$ & 0.565 \\
\hline Pulmonary disease & $7(12.5 \%)$ & $5(16.6 \%)$ & 0.523 \\
\hline \multicolumn{4}{|l|}{ Origin of the tumor } \\
\hline Uterine cervix & $48(85.7 \%)$ & $11(36.7 \%)$ & 0.012 \\
\hline Vulvar & $2(3.6 \%)$ & $16(53.3 \%)$ & 0.034 \\
\hline Uterine body & $4(7.1 \%)$ & $2(6.7 \%)$ & 0.988 \\
\hline Ovarian & $2(3.6 \%)$ & $1(3.3 \%)$ & 0.967 \\
\hline \multicolumn{4}{|l|}{ Type of disease } \\
\hline Primary & $45(80.3 \%)$ & $18(60.0 \%)$ & 0.452 \\
\hline Recurrent & $11(19.7 \%)$ & $12(40.0 \%)$ & 0.634 \\
\hline
\end{tabular}

Table II. Patients' characteristics classified according to the nutritional status.

\begin{tabular}{|c|c|c|c|}
\hline Characteristics & Albumin level $<3.5 \mathrm{~g} / \mathrm{dl}$ (23 cases) & Albumin level $>3.5 \mathrm{~g} / \mathrm{dl}$ (63 cases) & $p$-Value \\
\hline \multicolumn{4}{|l|}{ Age } \\
\hline$<60$ years & $14(60.9 \%)$ & $42(66.7 \%)$ & 0.834 \\
\hline$>60$ years & $9(39.1 \%)$ & $21(33.3 \%)$ & 0.752 \\
\hline \multicolumn{4}{|l|}{ BMI } \\
\hline$<18 \mathrm{~kg} / \mathrm{m}^{2}$ & $14(60.9 \%)$ & $-(0 \%)$ & 0.0001 \\
\hline $18-30 \mathrm{~kg} / \mathrm{m}^{2}$ & $1(4.3 \%)$ & $45(71.4 \%)$ & 0.002 \\
\hline$>30 \mathrm{~kg} / \mathrm{m}^{2}$ & $8(34.8 \%)$ & $18(28.6 \%)$ & 0.232 \\
\hline \multicolumn{4}{|l|}{ Comorbidities } \\
\hline Hypertension & $8(34.8 \%)$ & $12(19.0 \%)$ & 0.556 \\
\hline Diabetes mellitus & $9(39.1 \%)$ & $5(7.9 \%)$ & 0.076 \\
\hline Pulmonary disease & $8(34.8 \%)$ & $4(6.3 \%)$ & 0.063 \\
\hline \multicolumn{4}{|l|}{ Origin of the tumor } \\
\hline Uterine cervix & $12(52.2 \%)$ & $47(74.6 \%)$ & 0.531 \\
\hline Vulvar & $6(26.1 \%)$ & $12(19.0 \%)$ & 0.076 \\
\hline Uterine body & $3(13.0 \%)$ & $3(4.8 \%)$ & 0.565 \\
\hline Ovarian & $2(8.7 \%)$ & $1(1.6 \%)$ & 0.237 \\
\hline \multicolumn{4}{|l|}{ Type of disease } \\
\hline Primary & $20(86.9 \%)$ & $43(68.2 \%)$ & 0.056 \\
\hline Recurrent & $3(13.1 \%)$ & $20(31.8 \%)$ & 0.067 \\
\hline
\end{tabular}

pelvic reconstruction. Moreover, these procedures should be reserved for patients with a good clinical and biological condition $(13,14)$.

This fact was also demonstrated by the study conducted by Lyell et al. and published in 2019 in the American Journal of Surgery (15), which included 199 patients submitted to pelvic exenterations. Among these cases, perioperative complications were encountered in 177 patients within the first postoperative month, the most commonly encountered being represented by deep space/organ infection, chronic pain and wound infections. However, among these cases, reoperation was needed in 22 cases. Regarding the influence of the preoperative nutritional status, cases in which the preoperative level of the serum albumin was lower than 3.5 $\mathrm{g} / \mathrm{dl}$ reported a significantly higher rate of postoperative complications as well as a poorer rate of long-term survival (15). However, other authors consider that not albumin but prealbumin should be considered as a prognostic factor after extended surgical procedures due to the fact that pre-albumin has a shorter half-life than albumin (16). Even so, it seems 
in vivo $33: 2147-2152(2019)$

Table III. Intraoperative and postoperative outcomes stratified according to patients' age.

\begin{tabular}{|c|c|c|c|}
\hline Characteristics & Age $<60$ years ( 56 cases) & Age $>60$ years ( 30 cases $)$ & $p$-Value \\
\hline Time of surgery & $380 \mathrm{~min}$ & $220 \mathrm{~min}$ & 0.001 \\
\hline Estimated blood loss & $1500 \mathrm{ml}$ & $1100 \mathrm{ml}$ & 0.054 \\
\hline \multicolumn{4}{|l|}{ Urinary tract reconstruction } \\
\hline Continent conduit & $22(39.3 \%)$ & $2(6.7 \%)$ & 0.023 \\
\hline Incontinent conduit & $34(60.7 \%)$ & $28(93.3 \%)$ & 0.031 \\
\hline \multicolumn{4}{|l|}{ Digestive tract reconstruction } \\
\hline Anastomosis & $31(55.4 \%)$ & $3(10.0 \%)$ & 0.021 \\
\hline End colostomy & $25(44.6 \%)$ & $27(90.0 \%)$ & 0.043 \\
\hline Vaginal reconstruction & $14(25.0 \%)$ & $1(3.3 \%)$ & 0.0001 \\
\hline \multicolumn{4}{|l|}{ Resection margins } \\
\hline Negative & $49(87.5 \%)$ & $23(76.7 \%)$ & 0.565 \\
\hline Positive & $7(12.5 \%)$ & $7(22.3 \%)$ & 0.343 \\
\hline Length of hospital in stay - days & $14(10-42)$ & $22(12-61)$ & 0.034 \\
\hline Postoperative complications requiring reoperation & $14(25.0 \%)$ & $10(33.3 \%)$ & 0.561 \\
\hline Postoperative death (within 30 days) & $3(5.3 \%)$ & $2(6.7 \%)$ & 0.634 \\
\hline
\end{tabular}

Table IV. The impact of the serum albumin levels on the perioperative outcomes.

\begin{tabular}{|c|c|c|c|}
\hline Characteristics & $\begin{array}{c}\text { Albumin level }<3.5 \mathrm{~g} / \mathrm{d} l \\
\text { ( } 23 \text { cases })\end{array}$ & $\begin{array}{c}\text { Albumin level }>3.5 \mathrm{~g} / \mathrm{dl} \\
\quad(63 \text { cases })\end{array}$ & $p$-Value \\
\hline \multicolumn{4}{|l|}{ Urinary tract reconstruction } \\
\hline Continent conduit & $1(4.4 \%)$ & $23(36.5 \%)$ & 0.032 \\
\hline Incontinent conduit & $22(95.6 \%)$ & $40(63.5 \%)$ & 0.051 \\
\hline \multicolumn{4}{|l|}{ Digestive tract reconstruction } \\
\hline Anastomosis & $2(8.7 \%)$ & $32(50.8 \%)$ & 0.014 \\
\hline End colostomy & $21(91.3 \%)$ & $31(49.2 \%)$ & 0.033 \\
\hline Vaginal reconstruction & $2(8.7 \%)$ & $13(20.6 \%)$ & 0.045 \\
\hline Length of hospital in stay - days & $23(10-42)$ & $15(12-61)$ & 0.023 \\
\hline Postoperative complications requiring reoperation & $11(47.8 \%)$ & $13(20.6 \%)$ & 0.037 \\
\hline Postoperative death (within 30 days) & $3(13.0 \%)$ & $2(3.2 \%)$ & 0.056 \\
\hline
\end{tabular}

that malnutrition (defined by a poorer level of total serum proteins, albumin or pre-albumin) represents a significantly poor prognostic factor on the early postoperative outcomes after such extended procedures (17-19).

Another clinical factor which was widely studied in patients with pelvic exenteration is related to patient's age at the time of surgery. However, conflicting results have been reported so far. Therefore, it seems that age should not be considered as a formal contraindication for pelvic exenteration, the indication for surgery being rather tailored according to the other associated comorbidities $(20,21)$. In the study conducted by Huang et al. on 161 patients submitted to pelvic exenteration, the authors demonstrated that although associated comorbidities such as hypertension and pulmonary disease were more frequently encountered among elderly women, length of surgery, length of hospital stay and complication rates were similar between different age groups. However, the per cent of patients submitted to pelvic reconstructions, such as vaginal reconstruction and continent urinary reconstructions, was significantly lower among elderly patients, while the length of surgery was shorter in this category. The oncological degree of completeness - defined by the presence of negative resection margins and retrieved lymph nodes was similar between the different age groups (22).

Perineal reconstructions can be performed during the same surgical procedure or as a second step procedure, after resection and can consist of simple skin grafts or more demanding procedures such as myocutaneous or fasciocutaneous flaps depending on the dimensions of the defect, patient's medical history, local anatomy and associated comorbidities (23).

Other recent modifications, which have been implemented in the surgical technique of pelvic exenteration, are related to urinary tract and digestive tract reconstructions, improving in this way the functional outcomes of the patients as well as their psychological condition and their quality of life. However, association of such procedures requires in most of 
the cases more demanding procedures, longer operative times, higher amounts of blood loss and higher risks of postoperative complications. In consequence, the association of these reconstructive procedures should be reserved for patients with a good clinical and biological condition. Moreover, in cases where a recent history of pelvic radiation therapy exists, the risk of anastomotic leaks can increase up to $70 \%$, therefore reconstructions in such cases should be performed in highly selected cases and in high volume centers (13).

An interesting article which studied the permanent changes regarding the resected compartments and the reconstructive patterns after pelvic exenteration has been recently published by Waters et al. (24). The study included patients submitted to pelvic exenteration among three periods - 1988-2004 (193 cases), 2005-2010 (250 cases) and 20112018 (265 cases). Although age and co-morbidities were similar among the three study groups, the number of cases submitted to surgery for recurrent disease, the number of retrieved nodes as well as the number of complex resections involving the sacrum increased in the latter period. However, the number of cases which were submitted to exenterative surgery for gynecological malignancies decreased over time. When it comes to the type of reconstruction, although the perineal reconstructions using muscular flaps remained constant over the three periods, the number of cases submitted to urinary continent reconstructions increased over time. An increased rate of perioperative complications such as anastomotic leaks was reported during the second period of the study due to the fact that it was exactly the moment when the reconstructive procedures began to be widely included. Once more, experience was gained and the anastomotic leak rates decreased, a fact which was also observed in the last period of the study (24).

\section{Conclusion}

Pelvic exenteration remains a demanding procedure, associated with high rates of perioperative complications. Association of a reconstructive phase might increase the risk of perioperative complications, especially in elderly patients, with a poorer nutritional status as well as in obese patients. Therefore, such reconstructions should be indicated only in highly selected cases.

\section{Conflicts of Interest}

The Authors have no conflicts of interest to declare regarding this study.

\section{Authors' Contributions}

NB: Performed surgical procedures; IB, MV: prepared the manuscript; IB, MV, SD: data analysis, prepared the tables; MV, IB: part of the surgical team; IB: advised about surgical oncology procedure, revised the final draft of the manuscript.

\section{Acknowledgements}

This work was supported by the project entitled „Multidisciplinary Consortium for Supporting the Research Skills in Diagnosing, Treating and Identifying Predictive Factors of Malignant Gynecologic Disorders", project number PN-III-P1-1.2-PCCDI2017-0833.

\section{References}

1 Brunschwig A: Complete excision of pelvic viscera for advanced carcinoma; a one-stage abdominoperineal operation with end colostomy and bilateral ureteral implantation into the colon above the colostomy. Cancer 1: 177-183, 1948. PMID: 18875031. DOI: 10.1002/1097-0142(194807)1:2<177::aid-cncr2820010 203>3.0.co;2-a

2 Dindo D, Demartines N and Clavien PA: Classification of surgical complications: a new proposal with evaluation in a cohort of 6336 patients and results of a survey. Ann Surg 240: 205-213, 2004. PMID: 15273542. DOI: 10.1097/01.sla.0000133083.54934.ae

3 Hockel $\mathrm{M}$ and Dornhofer $\mathrm{N}$ : Pelvic exenteration for gynaecological tumours: achievements and unanswered questions. Lancet Oncol 7: 837-847, 2006. PMID: 17012046. DOI: $10.1016 / \mathrm{S} 1470-2045(06) 70903-2$

4 Palmer G, Martling A, Cedermark B and Holm T: A populationbased study on the management and outcome in patients with locally recurrent rectal cancer. Ann Surg Oncol 14: 447-454, 2007. PMID: 17139457. DOI: 10.1245/s10434-006-9256-9

5 Silberfein EJ, Kattepogu KM, Hu CY, Skibber JM, RodriguezBigas MA, Feig B, Das P, Krishnan S, Crane C, Kopetz S, Eng C and Chang GJ: Long-term survival and recurrence outcomes following surgery for distal rectal cancer. Ann Surg Oncol 17: 28632869, 2010. PMID: 20552409. DOI: 10.1245/s10434-010-1119-8

6 Magrina JF, Stanhope CR and Weaver AL: Pelvic exenterations: supralevator, infralevator, and with vulvectomy. Gynecol Oncol 64: 130-135, 1997. PMID: 8995561. DOI: 10.1006/gyno.1996.4532

7 Hockel M: Laterally extended endopelvic resection. Novel surgical treatment of locally recurrent cervical carcinoma involving the pelvic side wall. Gynecol Oncol 91: 369-377, 2003. PMID: 14599868. DOI: 10.1016/s0090-8258(03)00502-x

8 Wheeless CR Jr., Julian CG, Burnett LS and Dorsey JH: Synthetic pelvic floor sling to decrease small bowel complications after total exenteration. Obstet Gynecol 38: 779783, 1971. PMID: 5122480.

9 Schmitz RL, Schmitz HE, Smith CJ and Molitor JJ: Details of pelvic exenteration evolved during an experience with 75 cases. Am J Obstet Gynecol 80: 43-52, 1960. PMID: 14443165. DOI: $10.1016 / \mathrm{s} 0002-9378(16) 36415-8$.

10 Bacalbasa N, Tomescu D and Balescu I: Use of inflated foley catheters to prevent early empty pelvis complications following pelvic exenteration. Anticancer Res 35: 5543-5546, 2015. PMID: 26408723.

11 Disa JJ, Klein MH and Goldberg NH: Advantages of autologous fascia versus synthetic patch abdominal reconstruction in experimental animal defects. Plast Reconstr Surg 97: 801-806, 1996. PMID: 8628775. DOI: 10.1097/00006534-19960400000017

12 Said HK, Bevers M and Butler CE: Reconstruction of the pelvic floor and perineum with human acellular dermal matrix and thigh flaps following pelvic exenteration. Gynecol Oncol 107: 578-582, 2007. PMID: 17919700. DOI: 10.1016/j.ygyno.2007.08.063 
13 Hockel M and Dornhofer N: Vulvovaginal reconstruction for neoplastic disease. Lancet Oncol 9: 559-568, 2008. PMID: 18510987. DOI: 10.1016/S1470-2045(08)70147-5

14 Fowler JM: Incorporating pelvic/vaginal reconstruction into radical pelvic surgery. Gynecol Oncol 115: 154-163, 2009. PMID: 19596395. DOI: 10.1016/j.ygyno.2009.05.040

15 Lyell NJ, Kitano M, Smith B, Gleisner AL, Backes FJ, Cheng G, McCarter MD, Abdel-Misih S and Jones EL: The effect of preoperative nutritional status on postoperative complications and overall survival in patients undergoing pelvic exenteration: A multidisciplinary, multi-institutional cohort study. Am J Surg 218: 275280, 2019. PMID: 30982571. DOI: 10.1016/j.amjsurg.2019.03.021

16 Beaton J, Carey S, Solomon MJ, Tan KK and Young J: Preoperative body mass index, 30-day postoperative morbidity, length of stay and quality of life in patients undergoing pelvic exenteration surgery for recurrent and locally-advanced rectal cancer. Ann Coloproctol 30: 83-87, 2014. PMID: 2485121. DOI: 10.3393/ac.2014.30.2.83

17 Bozzetti F, Gianotti L, Braga M, Di Carlo V and Mariani L: Postoperative complications in gastrointestinal cancer patients: the joint role of the nutritional status and the nutritional support. Clin Nutr 26: 698-709, 2007. PMID: 17683831 DOI: 10.1016/j.clnu.2007.06.009.

18 Hennessey DB, Burke JP, Ni-Dhonochu T, Shields C, Winter DC and Mealy K: Preoperative hypoalbuminemia is an independent risk factor for the development of surgical site infection following gastrointestinal surgery: a multi-institutional study. Ann Surg 252: 325-329, 2010. PMID: 20647925. DOI: 10.1097/SLA.0b013e3181e9819a

19 Kehlet $\mathrm{H}$ and Wilmore DW: Multimodal strategies to improve surgical outcome. Am J Surg 183: 630-641, 2002. PMID: 12095591. DOI: 10.1016/s0002-9610(02)00866-8.
20 Ike H, Shimada H, Yamaguchi S, Ichikawa Y, Fujii S and Ohki S: Outcome of total pelvic exenteration for primary rectal cancer. Dis Colon Rectum 46: 474-480, 2003. PMID: 12682540. DOI: 10.1007/s10350-004-6585-2

21 Hopkins MP and Morley GW: Prognostic factors in advanced stage squamous cell cancer of the cervix. Cancer 72: 2389-2393, 1993. PMID: 8402454. DOI: 10.1002/1097-0142(19931015) 72:8<2389::aid-cncr2820720816>3.0.co;2-r

22 Huang M, Iglesias DA, Westin SN, Fellman B, Urbauer D, Schmeler KM, Frumovitz M, Ramirez PT and Soliman PT: Pelvic exenteration: impact of age on surgical and oncologic outcomes. Gynecol Oncol 132: 114-118, 2014, PMID: 24262877. DOI: 10.1016/j.ygyno.2013.11.014

23 Knapstein PG, Friedberg V and Sevin BU: Reconstructive Surgery in Gynecology. New York: Thieme Medical Publishers; 1990.

24 Waters PS, Peacock O, Warrier SK, Wakeman C, Eglinton T, Lynch AC, Frizelle FA, Heriot AG and McCormick J: Evolution of pelvic exenteration surgery- resectional trends and survival outcomes over three decades. Eur J Surg Oncol 2019. PMID: 31303376. DOI: 10.1016/j.ejso.2019.07.015
Received August 5, 2019

Revised September 9, 2019 Accepted September 11, 2019 